\title{
Multiscale Evolution of Strain Localisation Mechanisms in AZ31B Magnesium and 7050 Aluminum Alloys at High Strain Rates
}

\author{
Francis Tetteh, Solomon Duntu and Solomon Boakye-Yiadom
}

York University, Toronto, Ontario, Canada

At high strain rates (HSR), structural metals are exposed to increasingly extreme conditions in applied pressures, strain-rates, strains and temperatures and are easily susceptible to strain localization, which results in the formation of Adiabatic Shear Bands (ASBs). This is a critical mode of deformation which occurs when metals are subjected to compact loading such as ballistic impact, explosives, forging, high speed machining and penetrations[1]. It is generally agreed that the initiation of strain localization and formation of ASBs are manifestations of damage in metallic materials subjected to HSR and large strains of deformation and may lead to catastrophic failure instantaneously[2]. The mechanism of ASB has been attributed to thermo-mechanical instability that occurs in the deforming material during plastic deformation resulting from thermal softening[2,3]. Also, under the combined actions of heat and strain, dynamic recovery and recrystallization may occur in the shear band during dynamic impact conditions[2,4]. Studies report that ASBs are triggered by local inhomogenities including temperature differences, geometric variations and presence of voids and inclusions in the microstructure[5, 6].

Shear band formation has been reported in many structural metals such as steel, Titanium and some Aluminium(Al) series[2-4,6]. In contrast, microstructural evolutions of shear bands in AZ31B Magnesium(Mg) and $7050 \mathrm{Al}$ alloys are rarely reported. Recently, the interest in the deployment of light weight structural components has increased immensely in the aerospace and automobile industries. Both $\mathrm{Al}$ and $\mathrm{Mg}$ alloys are potential and competitive candidates(with $\mathrm{Mg}$ being 30 \% lighter than $\mathrm{Al}$ ) with low densities and high specific strength which makes them attractive in applications where their increased use leads to weight and energy savings[7]. However, in structural applications, both of these materials become susceptible to ASB formation which may lead to crack initiation and propagation and final failure. In order to effectively utilize these materials in applications where they will be subjected to HSR loading conditions, it is critical to understand their deformation under such conditions. Also, because strain gauges are not attached directly to specimens during testings, evolution of nucleation sites of strain localisations are not fully captured. Hence, the microstructural evolution of ASB initiations as well as strain field of plastic localisations resulting into ASB formations are required to determine the structural integrity of $\mathrm{Mg}$ and $\mathrm{Al}$ alloys under HRS conditions. Consequently, the aim of this work is to simultaneously study the microstructural evolution of ASB and in-situ meso-scale fields of strain localisations of AZ31B Mg and $7050 \mathrm{Al}$ alloys in order to compare and understand their mechanisms of ASB nucleation's.

A T6 tempered AZ31B Mg and Al 7050 alloys were impacted at room temperature using the direct impact hopkinson pressure bar at strain rates of $2031 \mathrm{~s}^{-1}$ and $1710 \mathrm{~s}^{-1}$ respectively. Digital image correlation(DIC) techniques was employed simultaneously using high speed cameras to monitor the nucleation sites of strain localisations.

It is observed that, at HSR compression loading, ASB evolved in both Mg and Al despite the brittle nature of $\mathrm{Mg}$. The evolution of $\mathrm{ASB}$ in $\mathrm{Mg}$ is uniquely characterized by coalescence of micro voids to form micro-crack whereas micro-twins are highly favored at ASB surroundings as shown in Fig. 1(a). This has similarly been observed in [8]. However, in $7050 \mathrm{Al}$, the evolved ASB is also characterized with multiple nucleation's of arced shaped lenticular narrow bands(Fig. 1(b)). The deformed regions also appears highly 
strain with grain distortions compared with the bulk specimen(Fig. 1(b2)). The true stress-true strain curve of $\mathrm{Mg}$ exhibited a sigmoid shape at early stage of deformation as shown in Fig. 2(a1). This is typical of twinning dominated deformation in $\mathrm{Mg}$. In AZ31B Mg, twinning regions may reorient and contribute to strain hardening rate in the form of texture hardening and form dislocation cells by creating dislocationtwin interactions [8,9]. However, the true stress strain behavior of $7050 \mathrm{Al}$ exhibited a concave downward characteristics(Fig. 2(a2)). The curve exhibits sharp rise in yield stress followed by steadily rise in stress at large strain up to peak stress before decreasing to rest. In addition, the DIC analysis revealed multiple nucleation sites of strain localisations which joins to form an hour glass shape at $\sim 45^{\mathrm{o}}$ after deformation(Fig. 2b).

During dynamic deformations, the mechanisms of ASB formation in $\mathrm{Mg}$ occurs by micro-voids coalescence which later propagates to form micro-cracks. The shear deformation surroundings are dominated by micro-twins which raises the strain hardening rates of the material at high strain rates. Likewise, multiple nucleation's of arc shaped lenticular ASBs evolves in Al 7050 alloy with distorted grains compared with the bulk specimen. AZ31B Mg exhibits sigmoid shape at early deformation stage whiles $7050 \mathrm{Al}$ exhibits concave downward true stress strain curves characteristics for large plastic strains during plastic deformations. It is suggested that ASBs nucleates with multiple nucleation sites of strain localisations regardless of the material.

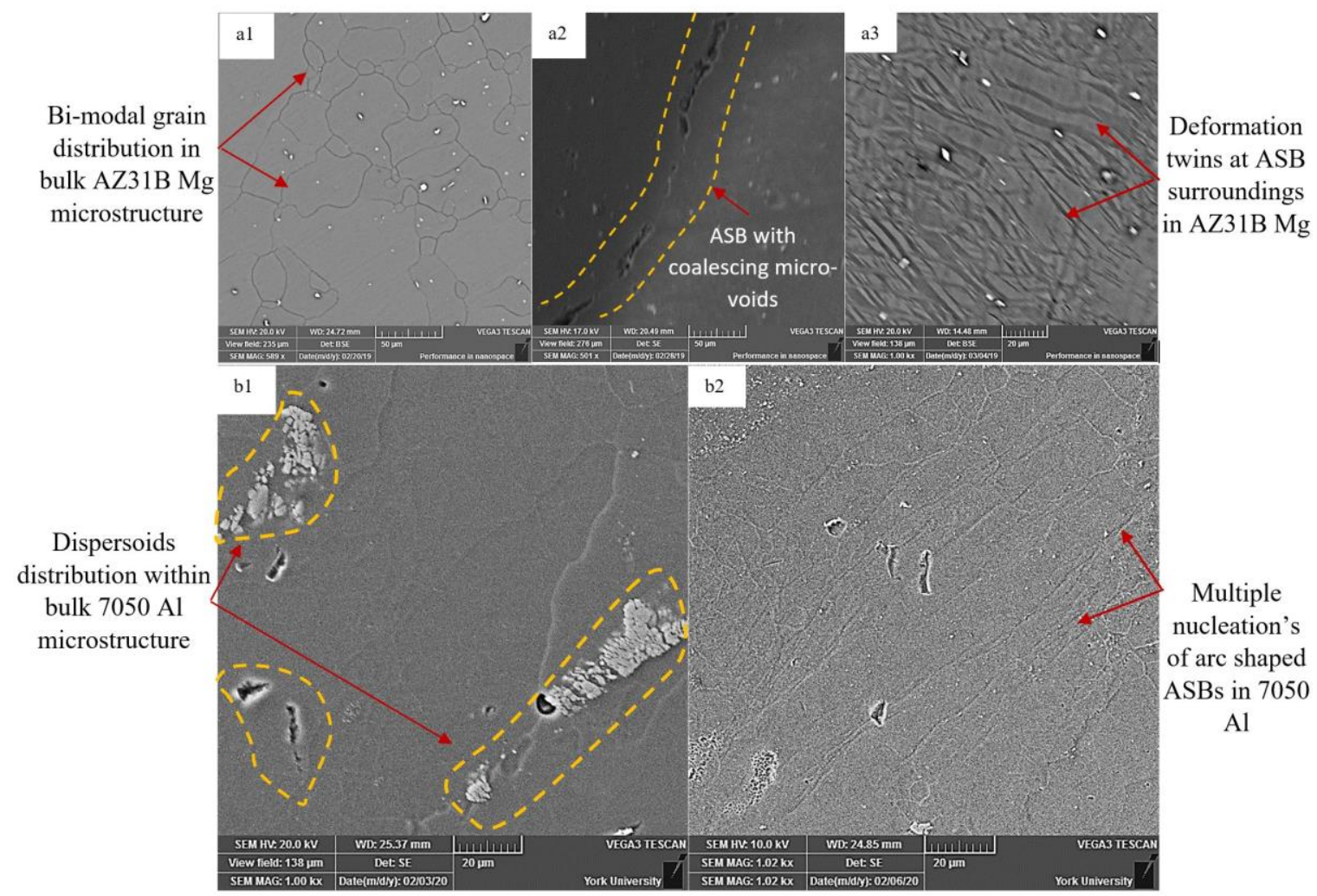

Figure 1. Scanning Electron Micrographs showing the microstructures of a1) bi-modal grain distribution in bulk AZ31B Mg, a2) micro-voids coalescence at ASB region in AZ31B Mg a3) deformation twins at ASB surroundings in AZ31B Mg b1) dispersids distribution in bulk 7050 Al alloy and b2) multiple ASB nucleations and grain distortions at highly strained regions in deformed $7050 \mathrm{Al}$ alloy. 


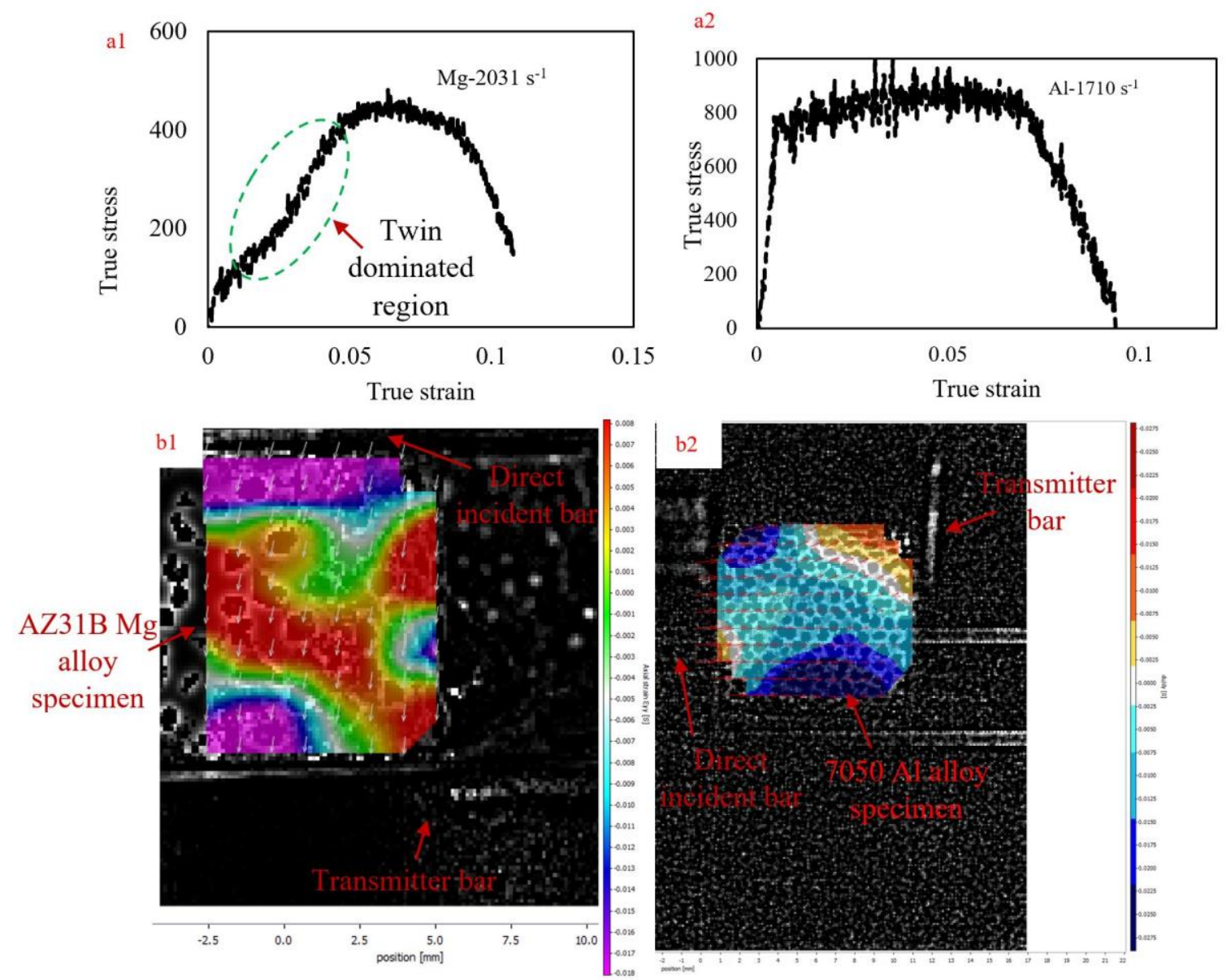

Figure 2. True stress strain behaviour of a) AZ31B Mg impacted at $2031 \mathrm{~s}-1$ and b2) $7050 \mathrm{Al}$ alloy impacted at $1710 \mathrm{~s}-1$, and Digital Image correlation analysis showing nucleation sites of strain localisations in b1) AZ31B Mg alloy and b2) $7050 \mathrm{Al}$ alloy.

References

1. H.C. Rogers, Annual Review Material Science, 9 (1979), p. 283-311.

2. D.H, Li et al., Material Science and Engineering A, 527 (2010), p. 3529-3535.

3. Y. Nie et al., Experimental Mechanics 60(2019), p.153-163.

4. S. Boakye Yiadom et al., Material Science and Engineering A, 615(2014), p.373-394.

5. A.M. Merzer, Journal of the Mechanics and Physics of Solids, 5 (1982), p. 323-338.

6. S.C Liao, J. Duffy, Journal of the Mechanics and Physics of Solids, 46(1998), p. 2201-2231.

7. B. Mordike, T. Ebert, Material Science and Engineering A, 302 (2001), p. 37-45

8. F. Tetteh and S. Boakye-Yiadom, Material Science and Engineering A, MSEA-D-19-03707R3 (2019).

9. N. Dixit, Acta Materialia, 87 (2015), p. 56-67. 